\title{
Serum Galectin-3 Levels Are Unlikely to Be a Useful Predictive Marker for Early-onset Preeclampsia Development
}

Asparuh Nikolov ${ }^{1}$, Nikola Popovski ${ }^{2}$, Alexander Blazhev ${ }^{3}$

${ }^{1}$ Scientific Division of Medicine, Institute for Scientific Research, Medical University of Pleven, Pleven, Bulgaria;

${ }^{2}$ Clinic of Obstetrics and Gynecology, University Hospital Pleven, Medical University of Pleven, Pleven, Bulgaria;

${ }^{3}$ Division of Biology, Medical University of Pleven, Pleven, Bulgaria

Received May 6, 2020; Accepted September 14, 2020.

Key words: Galectin-3 - Biomarker - Early-onset preeclampsia

Abstract: Galectin-3 (gal-3) is lectin which is presumed to interact with extracellular matrix proteins and cell surface glycoproteins in normal and pathophysiological conditions. The expression of gal-3 at the fetal-maternal interface partially overlaps that of gal-1, suggesting that an interplay between them might be important for hypertensive disorders in pregnancy like preeclampsia. The aim of our study was to test the hypothesis whether galectin-3 could be used as a predictive marker for early-onset preeclampsia development. 32 patients with early-onset preeclampsia were examined, mean age $28.8 \pm 5.5$; and 22 age matched normal pregnancies mean age $28.5 \pm 6.0$. The enzyme-linked immunosorbent assay (ELISA) was used for measuring serum galectin-3 levels. There were no significant differences between serum levels of galectin-3 in sera of preeclampsia patients compared to normal pregnant women - $14.1 \pm 4.77$ vs. $15.7 \pm 5.95 \mathrm{ng} / \mathrm{ml}(\mathrm{p}>0.05)$. Serum galectin-3 levels correlated with maternal age $(r=0.33 ; p=0.03)$ and $B M I$ (body mass index) $(r=0.52 ; p=0.01)$. Our data suggest that determination of serum galectin- 3 levels may not be a useful method for prediction of early-onset preeclampsia. Studies should be aimed to other categories of biomarkers.

This study was supported by the Centre of Scientific Research of Medical University, Pleven, Bulgaria.

Mailing Address: Assoc. Prof. Asparuh Nikolov, MD., PhD., Scientific Division of Medicine, Institute for Scientific Research, Medical University of Pleven, St. Kliment Ohridski Str., 5800 Pleven, Bulgaria; Phone: ++359 887100 672; e-mail: a_nicoloff@yahoo.com 


\section{Introduction}

Preeclampsia (PE) has not been fully studied yet. It is a major cause of maternal and perinatal morbidity and mortality (Gallo et al., 2016). PE and gestational hypertension $(\mathrm{GH})$ are associated with preterm delivery, intrauterine fetal death and increased maternal morbidity/mortality rate (Villar et al., 2006). Currently many biomarkers for early detection and prognosis of preeclampsia are investigated. The most widely used markers in clinical practice are: placental growth factor (PLGF) (Tsiakkas et al., 2015; Sung et al., 2017) and serum pregnancy-associated plasma protein-A (PAPP-A) (Kalousová et al., 2014; Patil et al., 2014). Unfortunately, their sensitivity and specificity is low yet. There is growing evidence that galectins are involved in pathophysiology of preeclampsia. However, preeclampsia does not always clinically present when remodelling has failed to occur (Karumanchi and Granger, 2016). Biomarkers of early preeclampsia detection are critical for risk stratification and testing therapies (Ferguson et al., 2017). Therefore, there is an urgent need for high quality, novel research in PE biomarkers, so that the best predictive strategy can be identified in order to improve the management of women in high risk.

Galectin-3 is an intra- and extracellular lectin. It is presumed to have an interaction with extracellular matrix proteins and cell surface glycoproteins. Currently, the physiological functions of this protein are in intensive studies. Ochieng et al. (1998) have established that recombinant human galectin-3 is a novel substrate for matrix metalloproteinases-2 and -9 (MMP-2 and MMP-9). Results from their study suggest that cleavage of galectin-3 by metalloproteinases alters the lectin's carbohydrate domain recognition. This process helps the tight glycoconjugates' binding. In result galectin molecules self-association is reduced.

"Galectin-3 is observed in fibroblasts, chondrocytes, osteoblasts, osteoclasts, keratinocytes, Schwann cells and gastric mucosa. It is also found in endothelial cells in a number of tissues, and in immune cells such as neutrophils, eosinophils, basophils, mast cells, Langerhans cells and dendritic cells (cell types reviewed in). It plays a role in adipocyte proliferation, and obese mice have more galectin-3 in adipocytes than lean subjects have. Galectin-3 can be both antiapoptotic and proapoptotic inductor. Galectin-3's potential therapeutic use is in large focusing on inhibition, because gal-3 promotes cancer and metastasis, and inhibition would hence have a great potential for therapeutic anti-cancer treatment" (Brinchmann et al., 2018).

An interesting difference between galectin- 1 and galectin- 3 is that galectin- 3 is considered a pro-inflammatory signal targeting various innate immune aspects (i.e. macrophages, mast cells, and neutrophils) to promote their activation, degranulation, and cytokine production (Alves et al., 2010).

Consistent with the confirmed antagonistic effects of galectin-1 and galectin- 3 on T-cell responses in vitro, ovine placental galectin-3 has been observed to promote T-cell proliferation and activation (Iglesias et al., 1998). In mice, uterine galectin-3 expression is selectively upregulated during early stages of pregnancy and localizes 
to the luminal and glandular epithelia and the primary decidual zone, whereas at later stages this lectin is predominantly expressed in the placenta (Phillips et al., 1996). This distribution, collectively with the lowered implantation rates discovered upon tissue-specific galectin-3 knock-down in the uterus (Yang et al., 2012), suggests the pivotal function of this lectin during the embryo-maternal crosstalk driving implantation. Galectin-3 well-shows a comparable spatio-temporal distribution throughout pregnancy, being upregulated in the endometrium at the peri-implantation duration and detected in the placental villous cytotrophoblasts; and extravillous trophoblasts lineages as gestation progresses (Blois and Barrientos, 2014).

Despite the appreciable characterization in several species, the physiological features of galectin-3 expression at the fetal-maternal interface are unexplored yet. To this moment there is small number of studies investigating galectins and especially galectin-3 as a marker predicting the development of preeclampsia.

The available data from galectin-3 studies in aspect of its involvement in preeclampsia development so far are:

1) Elevations in serum galectin-3 levels with increments in insulin resistance-related parameters and lipid profiles reflect the possible contribution of gal-3 to the harmful effects of IR (insulin resistance) and dyslipidemia levels on women with PE;

2) Galectin-3 may be both an initiating factor in the pathophysiology of preterm prelabor rupture of membranes, a marker in the prediction, and a target of preventing strategies of preterm prelabor rupture of membranes.

Therefore, we decided to test the hypothesis whether galectin-3 could be used as a predictive marker for early-onset preeclampsia development.

\section{Material and Methods}

Patient population

All patients were residing in the vicinity of the University Hospital Pleven. Subjects' sera were taken from October 2019 to February 2020. All the procedures followed were in accordance with the ethical standards of the responsible committee on human experimentation (institutional or regional) and with the Helsinki Declaration of 1975, as revised in 2000. Approval of local Ethics Committee was obtained and informed consent from adult research participants was obtained too. The study group consisted of 32 patients with preeclampsia, mean age $28.8 \pm 5.5$; and 22 age matched normal pregnancies mean age $28.5 \pm 6.0$ (Table 1 ).

\section{Study inclusion criteria}

- Pregnant women with clinical symptoms and laboratory criteria for early-onset preeclampsia (preeclampsia between 20 and 34 gestational week)

- Maintaining a current diet and exercise during the study

- Lack of metabolic syndrome and/or diabetes mellitus 


\section{Table 1 - Clinical data of women with preeclampsia and normal pregnant women}

\begin{tabular}{lcc}
\hline & Preeclampsia & Normal pregnant women \\
\hline Maternal age & $28.8 \pm 5.5$ & $28.5 \pm 6.0$ \\
BMI $\geq 25$ & $23 / 32^{*}$ & $4 / 22$ \\
Gravida & $2(2)^{* *}$ & $3(2)^{* *}$ \\
Parity & $1(2)^{* *}$ & $1(2)^{* *}$ \\
SBP $(m m ~ H g)$ & $138 \pm 17^{*}$ & $116 \pm 14$ \\
DBP (mm Hg) & $81 \pm 10^{*}$ & $68 \pm 8$ \\
Past history of preeclampsia & $1 / 32$ & $8 / 22^{*}$ \\
Family history of hypertension & 11 & $19^{*}$ \\
Chronic hypertension & $1 / 32$ & $10 / 22^{*}$ \\
Abnormal UAD & $18 / 32^{*}$ & $2 / 22$ \\
Smoker & $6 / 32$ & $2 / 22$ \\
Galectin-3 (ng/ml) & $14.1 \pm 4.77$ & $15.7 \pm 5.95$ \\
Number & $(\mathrm{n}=32)$ & $(\mathrm{n}=22)$ \\
\hline
\end{tabular}

BMI - body mass index; SBP - systolic blood pressure; DBP - diastolic blood pressure; UAD - uterine artery Doppler; data are shown as the mean \pm SD (standard deviation); ${ }^{*}<<0.05 ; * *$ data are expressed as median (interquartile range)

- Parameters of hypertension

- Presence/absence of risk factors

- Signed informed consent approved by the Ethics Committee at the University Hospital "G. Stranski" and the Medical University of Pleven

Study exclusion criteria:

- Diabetes mellitus

- Kidney disease

- Cardiovascular disease

- Signs of chorioamnionitis

- Presence of a fetus with chromosomal abnormality

Diagnostic criteria for preeclampsia

Preeclampsia is defined by hypertension in pregnancy* and coexistence of one or more of the following new-onset conditions**:

*Definition and classification of hypertension in pregnancy according to 2018 ESC guidelines for the management of cardiovascular diseases during pregnancy:

The definition of hypertension in pregnancy is based only on office (or in-hospital) $B P$ values (systolic BP $[S B P] \geq 140 \mathrm{~mm} \mathrm{Hg}$ and/or DBP $\geq 90 \mathrm{~mm} \mathrm{Hg}$ ) and distinguishes mildly (140-159/90-109 mm Hg) or severely ( $\geq 160 / 110 \mathrm{~mm} \mathrm{Hg}$ ) elevated BP, in contrast to the grades used by the joint ESC/ESH Hypertension Guidelines. 
Definition of early-onset and late onset preeclampsia according ISSHP (International Society for the study of Hypertension in Pregnancy)

Early-onset preeclampsia (EOP) is defined before 34 weeks' gestation, and late-onset preeclampsia (LOP) is defined after 34 weeks or later.

\section{**Proteinuria}

Spot urine protein/creatinine $>30 \mathrm{mg} / \mathrm{mmol}(0.3 \mathrm{mg} / \mathrm{mg})$ or $>300 \mathrm{mg} /$ day or at least $1 \mathrm{~g} / \mathrm{l}$ (" $2+$ ") on dipstick testing.

**Other maternal organ dysfunctions

1) Renal insufficiency (creatinine $>90 \mu \mathrm{mol} / \mathrm{l} ; 1.02 \mathrm{mg} / \mathrm{dl}$ )

2) Liver involvement (doubling of serum transaminases and/or severe right upper quadrant pain)

3) Neurological complications (eclampsia, altered mental status, blindness, stroke, or more commonly hyperreflexia when accompanied by clonus and severe headaches when accompanied by hyperreflexia and persistent visual scotomata)

4) Hematological complications (platelet count $<150,000 / \mathrm{dl}$, DIC, and hemolysis)

**Uteroplacental dysfunction

Fetal growth restriction

Blood pressure measurement

Arterial blood pressure was measured using a standard anearoid sphygmomanometer, to the nearest $2 \mathrm{~mm} \mathrm{Hg}$, in the dominant arm after at least 10 -min rest in supine position. Blood pressure measuring was performed by "Riester" blood pressure measuring tool - type -Precisa ${ }^{\circledR} \mathrm{N} ; \varnothing 64 \mathrm{~mm}$ aluminium, single-tube, cotton hook cuff, adult, No. 1362-104.

\section{Electrocardiography}

Electrocardiography (ECG) was performed for LVH (left ventricular hypertrophy) assessment (Sokolow-Lyon index $>35 \mathrm{~mm}$, or $\mathrm{R}$ in a $\mathrm{VL} \geq 11 \mathrm{~mm}$; Cornell voltage duration product $>2,440 \mathrm{~mm} / \mathrm{ms}$, or Cornell voltage $>28 \mathrm{~mm}$ in men or $>20 \mathrm{~mm}$ in women). ECG was performed by 12 -lead ECG machine "Fukuda" - type FX 8322.

Doppler of the uterine artery

Flow velocity waveforms of the uterine artery were performed by ultrasound apparatus using an AB 2-7 MHz convex abdominal probe (GE Voluson E10, GE Medical Systems, Zipf, Austria). Patients were evaluated once in the semirecumbent position by a single operator after bed rest for 5 minutes. Three consecutive waveforms were obtained in the Doppler study. The mean pulsatility index (PI) was 
calculated. An abnormal Doppler of uterine artery result was diagnosed as a mean $\mathrm{PI}>$ the $95^{\text {th }}$ percentile for each gestational age.

Enzyme-linked immunosorbent assay (ELISA)

It was used for measuring galectin-3 levels. Gal-3 levels were measured in serum samples using enzyme-linked immunosorbent assay (Galectin-3 Quantikine ELISA Kit R and D Systems, Minneapolis, MN, RD-DGAL30) according to the manufacturer's instructions.

\section{Statistical analyses}

The research data was processed with the computer programs Excel (Microsoft Corporation, Redmond, WA) and Statgraphics Plus (Manugistics, Rockville, MD) for Windows. All results were described in tables, graphs, numerical values (mean $\pm \mathrm{SD}$, share indicators and correlations). The level of significance was determined as $(p<0.05)$. The one-way analysis of variance with $F$-test in the ANOVA table was used for means comparison. K-W (Kruskal-Wallis) test was performed in cases with different from normal distribution. Median (M) was used in K-W test, together with first and third quartile Q1 and Q3 (twenty-fifth and seventy-fifth percentile P25 and 75P). The Pearson test was used for assessing of correlations. Multivariate regression analysis was also performed.

\section{Results}

There were no significant differences between serum levels of galectin- 3 in preeclampsia women compared to normal pregnant women $-14.1 \pm 4.77$ vs. $15.7 \pm 5.95 \mathrm{ng} / \mathrm{ml}(p>0.05)$ (Figure 1). Serum galectin-3 levels correlated with maternal age $(r=0.33 ; p=0.03)$ and BMI (body mass index) $(r=0.52 ; p=0.01)$.

\section{Discussion}

Preeclampsia is a common pregnancy complication. It is associated with high maternal morbidity and mortality rates and intrauterine foetal growth restriction.

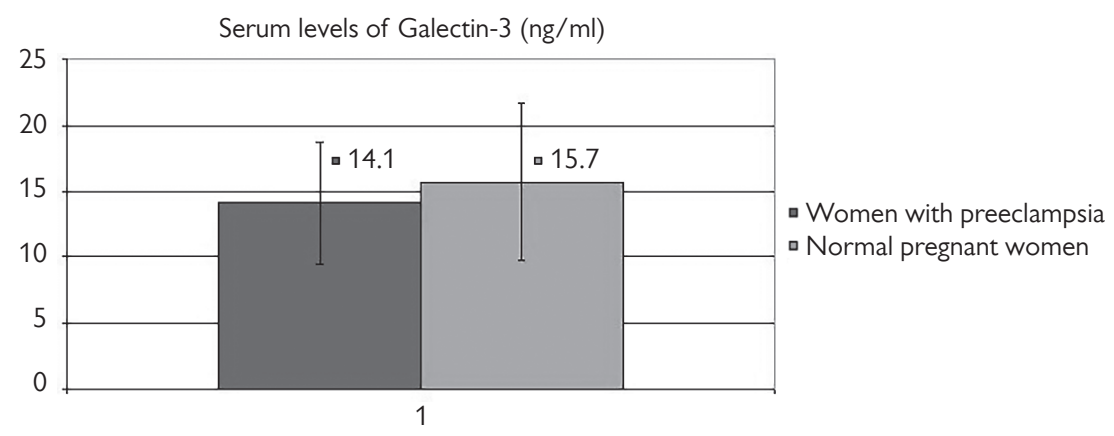

Figure 1 - Serum levels of galectin-3 in sera of women with preeclampsia and in normal pregnancies. 
This condition is characterised by high blood pressure and significant amount of protein in urine. Early detection of preeclampsia is very important for patient's risk stratification and an adequate management. It is extremely important for clinicians to perform risk assessment of developing preeclampsia. "The ASPRE trial (Combined Multimarker Screening and Randomized Patient Treatment with Aspirin for Evidence-based Preeclampsia Prevention): aspirin from 12 weeks concluded that treatment with low-dose aspirin in women at high risk for preterm PE reduces substantially the incidence of this disease". That is why the Fetal Medicine Foundation recommends preeclampsia pharmacologic prevention by admitting a prophylactic low-dose acetylsalicylic acid per os $(150 \mathrm{mg} /$ day $)$ in high risk women (Rolnik et al., 2017).

Hirashima et al. (2018) evaluated whether the serum level of galectin-1 (gal-1) at 18-24 and 27-31 weeks of gestation is a risk factor for predicting the later occurrence of not only preeclampsia (PE) but also gestational hypertension (GH). They measured serum levels of soluble fms-like tyrosine kinase 1 (sFlt-1), placental growth factor (PIGF), and gal-1 using an enzyme-linked immunosorbent assay in normal pregnant women, women with a later onset of $\mathrm{GH}$, and women with a later onset of PE at 18-24 and 27-31 weeks, respectively. Authors also determined gal-1 in 33 women with $\mathrm{GH}$ and women with PE after the onset. The levels of gal-1 after the onset of $\mathrm{GH}$, late-onset PE (onset at $\geq 34$ weeks), and early-onset PE (onset at $<34$ weeks) were significantly higher than those in normal pregnant women at 27-31 weeks. Authors' data show that, the serum level of gal-1 is a novel risk factor for both $\mathrm{GH}$ and PE, specifically its expression at a low level in the second trimester and a high level after onset.

Taha et al. (2020) study aimed to determine the correlations amongst serum apelin and galectin-3 levels and insulin resistance (IR) in women with PE. Authors made conclusion, that elevations in serum galectin-3 levels with increments in IR-related parameters and lipid profiles reflect the possible contribution of galectin-3 to the harmful effects of IR and dyslipidemia levels on women with PE.

Kaya et al. (2020) investigated maternal serum galectin-1 and galectin-3 levels in pregnancies complicated with preterm prelabor rupture of membranes (PPROM) and compared it with pregnancies delivered at term. Patients complicated with PPROM had significantly higher levels of galectin-1 and galectin-3 than the control group. Maternal serum galectin-3 levels were found significantly negatively correlated with the gestational age at delivery and birth weight. Maternal serum galectin-1 and galectin-3 levels were significantly higher in pregnancies complicated with PPROM. Galectin-1 and galectin-3, with their regulatory effects in key biological processes, may be both an initiating factor in the pathophysiology of PPROM, a marker in the prediction, and a target of preventing strategies of PPROM.

These studies (Hirashima et al., 2018; Kaya et al., 2020; Taha et al., 2020) have reported that galectins are involved in pathophysiology of preeclampsia. In our study we tested the hypothesis whether galectin-3 could be used as a biomarker predicting 
early-onset preeclampsia development. Our results did not support the hypothesis. We report, using the latest study cohort to date, that serum galectin-3 levels are unlikely to be a useful marker of early-onset preeclampsia development.

Possible explanations could be:

- The expression of galectin-3 at the fetal-maternal interface partially overlaps that of galectin-1, suggesting that an interplay between these lectins might be important for developmental processes and immune modulation during early pregnancy.

- It is known that galectin-3 can have both antiapoptotic and proapoptotic properties depending on its association with intracellular or extracellular binding partners. Additionally, this lectin appears to play an important role in the modulation of adaptive immunity, as it has been shown that galectin-3 can either promote or inhibit T-cell apoptosis.

- Unlike galectin-1, galectin-3 is considered a pro-inflammatory signal targeting diverse innate immune components (i.e. macrophages, mast cells, and neutrophils). Probably pregnancy promote their activation, degranulation, and cytokine production in a different way, which need further investigations.

- Galectin-3 interacts mainly with matrix metalloproteinase-2 and matrix metalloproteinase-9, but not with matrix metalloproteinase-1, which is known to be involved in the breakdown of components of extracellular matrix. Specifically, matrix metalloproteinase-1 breaks down the interstitial collagens, types I, II, and III.

- Galectin-3 expression is up regulated in the very early stages of preeclampsia, even before 20 weeks of gestation. The inclusion criteria for our study was pregnant women with early-onset preeclampsia (preeclampsia between 20 and 34 gestational week).

\section{Conclusion}

Serum galectin-3 levels are unlikely to be a useful marker of early-onset preeclampsia development. Studies should be aimed to other categories of biomarkers.

\section{References}

Alves, C. M., Silva, D. A., Azzolini, A. E., Marzocchi-Machado, C. M., Carvalho, J. V., Pajuaba, A. C., LucisanoValim, Y. M., Chammas, R., Liu, F. T., Roque-Barreira, M. C., Mineo, J. R. (2010) Galectin-3 plays a modulatory role in the lifespan and activation of murine neutrophils during early Toxoplasma gondii infection. Immunobiology 215, 475-485.

Blois, S., Barrientos, G. (2014) Galectin signature in normal pregnancy and preeclampsia. J. Reprod. Immunol. 102, 127-134.

Brinchmann, M. F., Patel, D. M., Iversen, M. H. (2018) The role of galectins as modulators of metabolism and inflammation. Mediators Inflamm. 2018, 9186940.

Ferguson, K. K., Meeker, J. D., McElrath, T. F., Mukherjee, B., Cantonwine, D. E. (2017) Repeated measures of inflammation and oxidative stress biomarkers in preeclamptic and normotensive pregnancies. Am. J. Obstet. Gynecol. 216(5), 5271-5279. 
Gallo, M., Wright, D., Casanova, C., Campanero, M., Nicolaides, K. (2016) Competing risks model in screening for preeclampsia by maternal factors and biomarkers at 19-24 weeks' gestation. Am. J. Obstet. Gynecol. 214(5), 6191-6197.

Hirashima, C., Ohkuchi, A., Nagayama, S., Suzuki, H., Takahashi, K., Ogoyama, M., Takahashi, H., Shirasuna, K., Matsubara, S. (2018) Galectin-1 as a novel risk factor for both gestational hypertension and preeclampsia, specifially its expression at a low level in the second trimester and a high level after onset. Hypertens. Res. 41(1), 45-52.

Iglesias, M. M., Rabinovich, G. A., Ambrosio, A. L., Castagna, L. F., Sotomayor, C. E., Wolfenstein-Todel, C. (1998) Purification of galectin-3 from ovine placenta: Developmentally regulated expression and immunological relevance. Glycobiology 8, 59-65.

Kalousová, M., Muravská, A., Zima, T. (2014) Pregnancy-associated plasma protein A (PAPP-A) and preeclampsia. Adv. Clin. Chem. 63, 169-209.

Karumanchi, S. A., Granger, J. P. (2016) Preeclampsia and pregnancy-related hypertensive disorders. Hypertension 67, 238-242.

Kaya, B., Turhan, U., Sezer, S., Kaya, S., Dağ, İ. (2020) Maternal serum galectin-1 and galectin-3 levels in pregnancies complicated with preterm prelabor rupture of membranes. J. Matern. Fetal Neonatal. Med. 33(5), 861-868.

Ochieng, J., Green, B., Evans, S., James, O., Warfield, P. (1998) Modulation of the biological functions of galectin-3 by matrix metalloproteinases. Biochim. Biophys. Acta 1379, 97-106.

Patil, M., Panchanadikar, T. M., Wagh, G. (2014) Variation of Papp-A level in the first trimester of pregnancy and its clinical outcome. J. Obstet. Gynaecol. India 64(2), 116-119.

Phillips, B., Knisley, K., Weitlauf, K. D., Dorsett, J., Lee, V., Weitlauf, H. (1996) Differential expression of two beta-galactoside-binding lectins in the reproductive tracts of pregnant mice. Biol. Reprod. 55, 548-558.

Rolnik, D. L., Wright, D., Poon, L. C., O'Gorman, N., Syngelaki, A. (2017) Aspirin versus placebo in pregnancies at high risk for preterm preeclampsia. N. Engl. J. Med. 377(7), 613-622.

Sung, K. U., Jeong, A. R., Kyung, J. E., Hyeok, K. E. (2017) Maternal serum placental growth factor and pregnancy-associated plasma protein A measured in the first trimester as parameters of subsequent pre-eclampsia and small-for-gestational-age infants: a prospective observational study. Obstet. Gynecol. Sci. 60(2), 154-162.

Taha, A. S., Zahraei, Z., Al-Hakeim, H. (2020) Serum apelin and galactin-3 in preeclampsia in Iraq. Hypertens. Pregnancy 2020, 1-8.

Tsiakkas, A., Duvdevani, N., Wright, A., Wright, D., Nicolaides, K. H. (2015) Serum placental growth factor in the three trimesters of pregnancy: Effects of maternal characteristics and medical history. Ultrasound Obstet. Gynecol. 45, 591-598.

Villar, J., Carroli, G., Wojdyla, D., Abalos, E., Giordano, D. (2006) Preeclampsia, gestational hypertension and intrauterine growth restriction, related or independent conditions? Am. J. Obstet. Gynecol. 194(4), 921-931.

Yang, H., Lei, C., Zhang, W. (2012) Expression of galectin-3 in mouse endometrium and its effect during embryo implantation. Reprod. Biomed. Online 24, 116-122.

Nikolov A.; Popovski N.; Blazhev A. 